\title{
The role of configuration and target discriminability in a visual search task*
}

\author{
JAMES R. POMERANTZ $\div$ and W. R. GARNER \\ Yale Lniversity, Vew Haren. Comnecticut 06510
}

\begin{abstract}
Ss sorted through decks of cards bearing five-element patterns, separating those in which all five were identical from those in which one of the five was different from the remaining four. Sorting time depended on the configuration and size of the pattern in which the elements were arranged but was independent of the goodness of these patterns. It was concluded that Ss did not attend to the patterns themselves, but just to the elements comprising them in searching for the discrepant element. The differences owing to the patterns appear to be due in part to the compactness of the configurations.
\end{abstract}

In many visual search tasks, it has been found that the amount of time required for an observer to locate a target in an array increases linearly with the number of nontarget elements that must be examined (Neisser, 1964; Atkinson et al, 1969). This finding has often been interpreted as supporting a serial search model in which the observer processes one element in the array at a time, decides if it is a target, and, if not, proceeds to the next element. With other visual search tasks, however, parallel processing of the array has been reported; that is, search time is largely independent of the number of elements in the array, indicating that all the elements are being examined simultaneously. The main distinction between these two types of tasks is that, in the latter, the nontargets are all identical. For example, the task might be to locate a triangle in a field of circles.

The most extensive investigations into this parallel search process have come from Volkmann and his associates $(1964,1965)$, little of whose research has appeared in the literature. They have concluded that for such tasks there exists an area of fast search, defined in terms of visual angle and centered about the fovea, within which stimulus elements are processed simultaneously, largely irrespective of their number or density. Stimulus elements lying outside this area require serial processing in the form of successive fixations. Moreover, they have found the extent of this area to be dependent on the particular discrimination required, constricting with more difficult ones. Support for their principal finding of a parallel search was reported by Donderi and Zelnicker (1969) and by Egeth, Jonides, and Wall (in press).

It is possible in a parallel search that the analyses of elements that are being performed simultaneously are not actually independent of one another and that

*This research was supported by Grant MH 14229 from the National Institute of Mental Health to Yale University.

$\div$ Requests for reprints should be sent to James R. Pomerantz, Department of Psychology, Yale University, New Haven, Connecticut 06510. interactions among the elements in the display might occur. Such a process would be parallel in the temporal sense but not in the operational sense. The question behind the present study is whether stimulus elements in the parallel search are processed separately as isolated units or whether interelement factors, especially higher order ones such as the configuration of the elements in the array, are important. If the observer encodes or otherwise attends to the pattern of the stimulus elements rather than simply dealing with the elements individually, then properties of patterns which affect performance in other visual tasks should influence the search process as well.

The configurational variable used in the experiments below was pattern goodness, although that concept as used here is operationally defined in terms of number of alternative stimuli. Garner (1962) has argued that the perception of a single stimulus is affected by the number of alternatives the stimulus has, i.e., the number of patterns that are equivalent to the stimulus pattern by some criterion. The size of this subset of alternative patterns determines the goodness or redundancy of the pattern: the smaller the subset, the greater the redundancy and the better the pattern (Gamer, 1970).

Several experiments relevant to the present study have revealed performance correlates of pattern goodness. Clement and Varnadoe (1967) and Clement and Weiman (1970), using the same type of five-element patterns used here, found that pairs of good patterns are discriminated more quickly than pairs of poor ones, even when $S$ knows that the discrimination can be made on the basis of a single element. Moreover, instructions to attend only to this critical element and to ignore the configuration as a whole were largely ineffectual. These studies suggest that processing of larger units may be mandatory, even when such processing is not logically required to perform the task. Checkosky and Whitlock's (in press) results from a Sternberg-type of recognition experiment indicate that poorer patterns take longer to be encoded than good ones. Thus, if $S$ s in a visual search task do respond to larger units or patterns and not just 


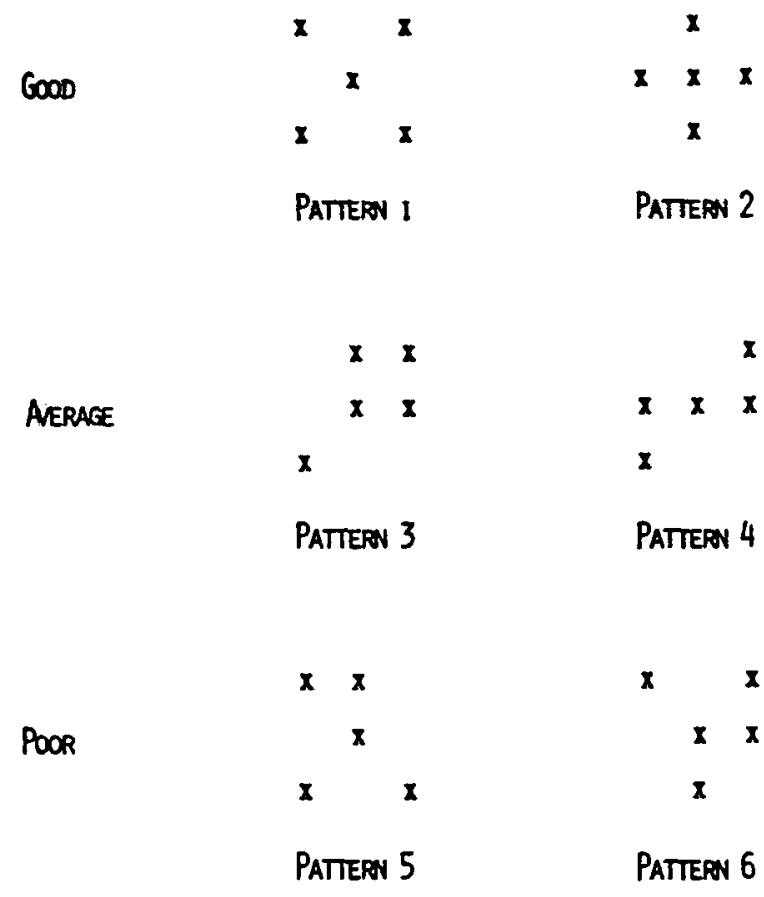

Fig. 1. The six patterns used in this study.

to individual elements, then the better patterns should be encoded more rapidly and the search process should be speeded accordingly.

In one visual search task, Krueger (1970) demonstrated that $S s$ can and do respond to larger patterns of elements, although his patterns were not ones of spatial configuration. He showed that a target letter could be located in a field of background letters more rapidly when these letters formed meaningful words than when they were arranged randomly. The redundancy of the word patterns presumably allowed them to be encoded more rapidly, thus accelerating the search process.

\section{PURPOSE OF STUDY}

In the present study, Ss searched through five-element arrays, deciding for each if all five elements were identical or if one differed from the others. The goodness of the patterns in which the elements were arrayed was varied to determine whether, in fact, the elements were being encoded as independent units or as unitary patterns. In addition to configuration itself, the size of the patterns was varied as well as the discriminability between the discrepant target element and the homogeneous background elements.

\section{EXPERIMENT I}

\section{Method}

\section{Stimuli}

The patterns used in this study were drawn from the five-element patterns first used by Garner and Clement (1963).
Briefly, these patterns are constructed by filling in five of the nine cells of an imaginary 3 by 3 square matrix. Six such patterns were used in these experiments, and they are shown in Fig. 1. The goodnes: of each pattern was defined by the number of equivalent patterns generated by mirror reflection and by rotation in increments of $90 \mathrm{deg}$. Patterns 1 and 2 were unique by this criterion: any reflection or unit rotation of these patterns yielded the same pattern. Thus, each was a one-alternative pattern and was termed "good." Patterns 5 and 6 , on the other hand, each had eight alternatives by this criterion and were called "poor." Patterns 3 and 4 had four alternatives each and were labeled "average."

The elements comprising these patterns were letters. The letters were typed on $6.3 \times 8.9 \mathrm{~cm}$ white cards, oriented vertically, in uppercase type with an IBM electric typewriter and carbon ribbon. The background or nontarget letters were Xs. The discriminability of the target element from the nontarget was varied: the target was either a "V" or an "O." In addition, the size of the patterns was varied by altering the spacing of the letters (but not the size of the letters themselves) by a factor of 2. The distance from midpoint to midpoint of adjacent cells in the imaginary 3 by 3 matrix was $4 \mathrm{~mm}$ for the small patterns and $8 \mathrm{~mm}$ for the large, while the letters themselves in both the large and small patterns measured approximately $2 \times 2 \mathrm{~mm}$. Thus, the major experimental variables of this study were pattern goodness, pattern size, and target discriminability.

\section{Procedure}

A speeded card-sorting technique was used to measure rate of visual search. Decks of 30 cards each were constructed, with each card bearing a single five-letter pattern. In each deck, 15 of the cards had patterns composed of five nontargets, while the remaining 15 had four nontargets and one target each. Within these latter 15 cards, the target occurred three times in each of the five possible positions in the pattern. There were 24 such decks in all, representing all combinations of the six patterns, two sizes, and two discriminabilities. The order of cards within decks was randomized by shuffling the decks five times in the presence of $S$ before each sort.

The S's task was to sort the decks of cards as quickly as possible into two piles, one for cards with targets and one for cards without targets. E gave $S$ a warning signal orally before activating a footswitch, which started a centisecond timer and served as a cue for $\mathrm{S}$ to begin sorting the deck. $\mathrm{S}$ was allowed to sort the cards as he saw fit; most Ss held the cards at a distance of about $30 \mathrm{~cm}$ from their eyes, yielding visual angles of $1 \mathrm{deg}$ $32 \mathrm{~min}$ and $3 \mathrm{deg} 4 \mathrm{~min}$ for the small and large patterns, respectively, measured from midpoint to midpoint of cells on opposite sides of the imaginary 3 by 3 matrix. These visual angles are well within Volkmann's hypothesized area of fast search.

Following each sort, $\mathrm{S}$ was informed of his sorting time, which $E$ recorded along with the number of errors made. if any. Each $S$ sorted all decks once in one order and then again in opposite order, with the sequence of decks counterbalanced across Ss. Ss were run individually for one 1.5 -h session with a 10 -min break after the first replication. Eight warm-up trials were given with similar stimuli at the beginning of the session.

\section{Subjects}

Of the 14 naive Ss ( 9 male) who served in the study. 13 were unpaid Yale undergraduates fulfilling a requirement of an introductory psychology course and 1 was a psychology graduate student who was paid.

\section{Results}

The results of the first experiment are shown in Table 1. Analysis of variance of sorting times revealed 
Table 1

Mean Sorting Time for the Six Patterns. Classified by (A) the Discrimination Required and (B) the Size of the Pattern*

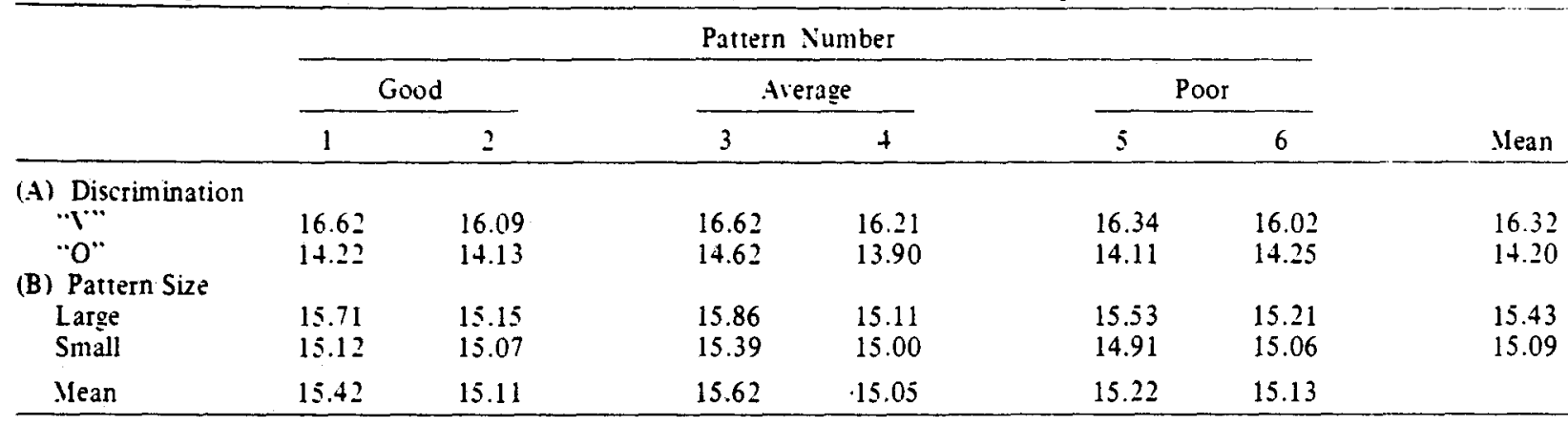

*The entries. based on $V^{*}=56$. are in seconds and represent the time required to sort a deck of 30 cards.

that the six patterns were a significant source of variance $[F(5.65)=4.08 . p<.01]$. However, a Newman-Keuls multiple comparison test of these six means showed that none of them differed significantly from any of the others. The hypothesis that sorting times would be related monotonically to the goodness of the patterns clearly was not supported. Indeed, the six means distributed themselves completely symmetrically with respect to the pattern goodness classification: the good patterns, 1 and 2, ranked fifth and second fastest in sorting time, respectively: the average patterns, 3 and 4 , ranked sixth and first; and the poor patterns, 5 and 6 , ranked fourth and third. This same overall rank ordering was shown by both the faster and the slower half of the Ss.

Table 1 shows these six means analyzed by target discriminability and by pattern size. The symmetrical distribution of the six means with respect to the pattern goodness classification is evident again in two of the four analyses, with the remaining two showing no consistent pattern of results. In short, it appears that pattern goodness had no effect on search speed.

Target discriminability made a difference: decks were sorted significantly faster when the target letter was an "O" than when it was a "V" $[F(1,13)=104.0, p<.01]$. The small patterns were searched more rapidly than the large ones $[F(1,13)=47.9, p<.01]$. Only the replications variable significantly interacted with patterns $[F(5,65)=2.7, p<.05]$, but replications did not interact at all with the pattern goodness dimension.

The error rate for the average $\mathrm{S}$ was well under $1 \%$, with the range for individual Ss extending from $0 \%$ to $2.2 \%$.

\section{Discussion}

The principal positive finding from the first experiment was that both pattern size and target discriminability are potent variables influencing search rate. However, while the pattern in which elements are placed in the array made a slight difference in the speed with which a discrepant target element was found, these small differences did not appear to be due to the goodness or redundancy of the patterns. Moreover, the absence of any significant interaction of patterns with the size or discriminability variables indicated that this relative insensitivity to configuration was fairly stable and was not due to an inappropriate choice of levels on these dimensions. Thus, this result would appear to reject the hypothesis that the entire pattern is encoded as a whole before the search process begins. Otherwise, differences in pattern goodness should have affected encoding time and so total time for the completion of the search.

There is one aspect of the experimental task which could have atteriuated processing differences among the patterns. Ss sorted through 30-card decks, within each of which the pattern of elements did nor vary. Rather, patterns varied only between decks. Similarly, the size of the configurations and the discrimination required did not vary within decks. Thus, the sorting of a particular deck involved exposure to the same pattern, size, and discrimination 30 times in a row. It is possible that this repetition itself facilitated the process of pattern encoding such that any prior differences in encoding time attributable to pattern goodness would be eliminated by a ceiling effect. To test whether this stimulus repetition was responsible for the absence of any effect of pattern goodness, a second experiment was conducted in which the stimulus pattern, along with size and target discriminability, varied within decks, with pattern goodness alone remaining constant.

\section{EXPERIMENT II}

\section{Method}

\section{Stimuli and Procedure}

The stimuli and procedure were the same as those in Experiment $I$. All that changed was the assignment of cards to decks. In Experiment I: for each level of pattern goodness there were eight decks of 30 cards each ( 2 patterns by 2 sizes by 2 discriminabilities), making a total of 240 cards. In Experiment II, these 240 cards were intermixed separately at each level of goodness. as thoroughly as possible, to create eight new decks, with each deck again containing 30 cards which now 
varied in size, discriminability, and pattern, but not in pattern goodness.

\section{Subjects}

Nine Ss were used (eight female), all of whom were college students recruited during the summer in New Haven, Connecticut. They were all paid $\$ 1.75$ to serve in a single $1-\mathrm{h}$ session.

\section{Results}

Sorting times were substantially slower in Experiment II, by almost $2.0 \mathrm{sec}$ on the average. Thus, any ceiling effect due to simple repetition in the first experiment would have been reduced or eliminated in Experiment II. Nevertheless, analysis of variance of sorting times indicated that pattern goodness again had no significant effect on sorting times $[F(2,24)<1]$. The actual means based on total sorting times for the 30 -card decks were as follows: $16.71 \mathrm{sec}$ for the good patterns, 17.21 for the average, and 17.07 for the poor patterns. Notice that this rank ordering of goodness levels by search speed is different from that obtained in the first experiment, where the poor patterns yielded the fastest sorting times, the good patterns were next fastest, and the average ones were the slowest. This lack of correspondence is a further indication that pattern goodness has no systematic effect on this type of search task.

\section{GENERAL DISCUSSION}

The results of Experiment II support the principal conclusion from the first experiment that the goodness of a pattern in which symbols are arranged has no regular effect upon the speed with which those symbols can be searched for a discrepant target element. Based on the assumption discussed above that better patterns should be encoded more rapidly than poor ones, this finding is interpreted to mean that Ss do not encode the pattern of elements as a whole before searching for the target element. Thus, the generalization from pattern discrimination studies that patterns are normally processed as whole entities, even when this is not required to perform the task, does not appear to apply to this visual search task.

\section{Area of Fast Search}

A model consistent with Volkmann's hypothesis of an area of fast search would hold that the search process consists of a simultaneous but independent examination of all elements in the array. A model of this sort would predict no differences in search time stemming from size or configurational differences in the arrays, provided that all patterns fall within the area of fast search. The first experiment of the present study showed that configurational differences did affect search time, although this effect was of small stature (and was independent of the pattern goodness dimension). Moreover, the size or density of the patterns was shown to have a highly reliable effect.

There are two probable reasons for this discrepancy. The first involves Volkmann's conclusion, which is corroborated in the present experiment, that the area of fast search shrinks with more difficult discriminations. If the letter discriminations required in the present study were more difficult than were the shape discriminations (e.g., circles vs triangles) in. Volkmann's work, then perhaps the present patterns exceeded the critical area for parallel search and so required some serial processing. Another somewhat simpler explanation for the discrepancy is that Volkmann's procedure controlled for fixation, which, of course, is not possible with the card-sorting technique used here. Since the area of fast search is centered about the fixation point, fixation of a point displaced from the center of a pattern could locate one or more of the elements outside of the parallel search area and thus necessitate some serial processing. The larger the pattern, the greater the probability of this situation occurring, so this reasoning could explain the differences in search rate between the two pattern sizes.

The small but significant differences among the patterns themselves found in the first experiment could be due to variations in the size or compactness of the six patterns. As a coarse index of compactness appropriate to these configurations, the distance from the geometric center to the most extreme element of each pattern was determined. Assuming that the locus of initial fixation is not constant but varies about the geometric center of the pattern (see, e.g., Richards \& Kaufman, 1969), this measure should correspond roughly to the relative probability of at least one element lying outside the area of fast search. The regression of the sorting time means for the six patterns on this measure was significant $[F(1,65)=7.52, p<.01]$ and accounted for $37 \%$ of the between-pattern variance. The residual variance not accounted for by this regression pattern was also significant, indicating that some other component of the configurations was affecting search rate. While it is likely that a more sophisticated measure of compactness would account for more of the differences between patterns, establishing the appropriateness of such a measure would require additional assumptions about the nature of the fast search and fixation processes.

\section{Parallel vs Serial Search}

One final point needs discussion. The conclusion that the pattern of the elements to be searched is only of indirect importance is limited just to the parallel searching found with homogeneous background elements. Pattern goodness might well be influential in the more familiar serial search through heterogeneous backgrounds. There are important differences between these two types of search, and indeed it is not clear what 
they have in common. In fact, it is possible that the parallel search is not really a search at all, in the usual sense of a hunt for a particular element, but is better characterized as a same-different judgment or even a figure-ground segregation process. The latter interpretation is particularly appealing in that figure-ground segregation has been hypothesized (Neisser. 1967) to be handled by a primitive preattentive process that acts in parallel. Should this interpretation prove correct, then it would appear that parallel searching and serial searching are quite different in nature and generalizations between the two would be accordingly speculative.

\section{REFERENCES}

Atkinson, R. C.. Holmgren, J. E., \& Juola, J. F. Processing time as influenced by the number of elements in a visual display. Perception \& Psychophysics, 1969, 6, 321-326.

Checkosky, S. F., \& Whitlock. P. The effects of pattern goodness on recognition time in a memory search task. Perception \& Psychophysics, in press.

Clement, D. E., \& Varnadoe, K. W. Pattern uncertainty and the discrimination of visual patterns. Perception \& Psychophysics, $1967,2.427-431$.

Clement, D. E., \& Weiman, F. R. Instructions, strategies, and pattern uncertainty in a visual discrimination task. Perception \& Psychophysics, 1970, 7, 333-336.
Donderi, D. C., \& Zelnicker. D. Parallel processing in visual same-different decisions. Perception \& Psychophysics. 1969. $5,197-200$.

Egeth. H., Jonides, J., \& Wall. S. Parallel processing of multielement displays. Cognitive Psychology. in press.

Garner, W. R. Uncertainty and stnicture as psichological concepts. New York: Wiley, 1962.

Garner, W. R. Good patterns have few alternatives. American Scientist, 1970, 58, 34-42.

Garner, W. R., \& Clement, D. E. Goodness of pattern and pattern uncertainty. Journal of Verbal Learning \& Verbal Behavior, 1963, 2, 446-452.

Krueger, L. E. Search time in a redundant visual display. Journal of Experimental Psychology, 1970, 83, 391-399.

Neisser, U. Visual search. Scientifíc American, 1964, 210, 94-102.

Neisser, U. Cognitive psychology. New York: AppletonCentury-Crofts, 1967.

Richards, W., \& Kaufman, L. "Center-of-gravity" tendencies for fixations and flow patterns. Perception \& Psychophysics, $1969,5,81-84$.

Volkmann, J., Corbin, H. H., Eddy, N. B., \& Coonley, C. The range of visual search. Technical Documentary Report No. ESD-TDR-64-535, November 1964, Mt. Holyoke College, Contract No. AF 19 (604)-3037, U.S. Air Force.

Volkmann, J., \& Corbin, H. H. Further experiments on the range of visual search. Technical Documentary Report No. ESD-TDR-65-169, January 1965, Mt. Holyoke College, Contract No. AF 19 (628)-2443, U.S. Air Force.

(Accepted September 12, 1972.) 